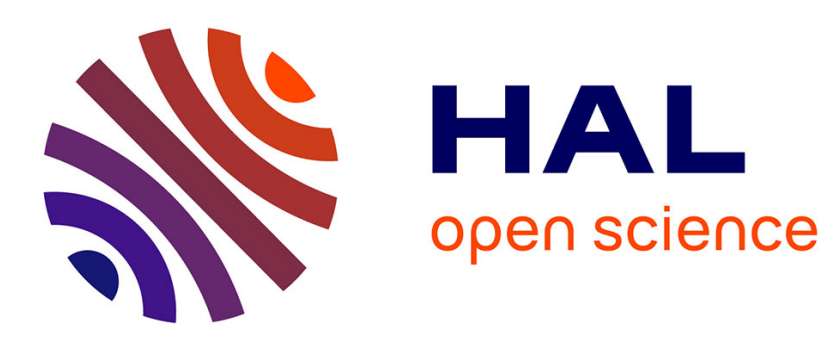

\title{
Experimental Evidence on the Influence of Extended Defects on a Structural Phase Transition: Polymer Chains in a Monomer Matrix
}

\author{
S. Longeville, J. Even, M. Bertault, F. Moussa
}

\section{- To cite this version:}

S. Longeville, J. Even, M. Bertault, F. Moussa. Experimental Evidence on the Influence of Extended Defects on a Structural Phase Transition: Polymer Chains in a Monomer Matrix. Journal de Physique I, 1997, 7 (10), pp.1245-1258. 10.1051/jp1:1997121 • jpa-00247452

HAL Id: jpa-00247452

https://hal.science/jpa-00247452

Submitted on 1 Jan 1997

HAL is a multi-disciplinary open access archive for the deposit and dissemination of scientific research documents, whether they are published or not. The documents may come from teaching and research institutions in France or abroad, or from public or private research centers.
L'archive ouverte pluridisciplinaire $\mathbf{H A L}$, est destinée au dépôt et à la diffusion de documents scientifiques de niveau recherche, publiés ou non, émanant des établissements d'enseignement et de recherche français ou étrangers, des laboratoires publics ou privés. 


\title{
Experimental Evidence on the Influence of Extended Defects on a Structural Phase Transition: Polymer Chains in a Monomer Matrix
}

\author{
S. Longeville $\left({ }^{1}\right)$, J. Even $\left({ }^{1}\right)$, M. Bertault $\left({ }^{1, *}\right)$ and F. Moussa $\left({ }^{2}\right)$ \\ $\left({ }^{1}\right)$ Groupe Matière Condensée et Matériaux $\left({ }^{* *}\right)$, Université de Rennes I, Campus de Beaulieu, \\ 35042 Rennes Cedex , France \\ $\left({ }^{2}\right)$ Laboratoire Léon Brillouin (CEA-CNRS), CE Saclay, 91191 Gif-sur-Yvette Cedex, France
}

(Received 5 June 1996, revised 8 April 1997, accepted 17 June 1997)

PACS.61.12.Ld - Single-crystal and powder diffraction

PACS.61.72.Dd - Experimental determination of defects by diffraction and scattering

PACS.61.50.Ks - Crystallographic aspects of phase transformations; pressure effects

\begin{abstract}
The influence of the polymerization on the static aspects of the structural phase transition in a monomer-polymer pTS-diacetylene system is studied by neutron scattering. The disorder arises probably from the frustration of the incommensurate modulation present in the pure monomer; the influence of the polymer chains acting as extended defects appears the determining factor in the observed phenomena.
\end{abstract}

Résumé. - Nous étudions les aspects statiques des transitions de phase dans un système mixte monomère-polymère par diffusion de neutrons. Le désordre provient probablement d'une frustration de la modulation incommensurable qui existe dans les cristaux de monomère pur. L'influence des défauts étendus que constituent les chaines de polymères est le facteur prépondérant qui apparait dans les phénomènes observés.

\section{Introduction}

Some diacetylenes $\left(\mathrm{R}-\mathrm{C} \equiv \mathrm{C}-\mathrm{C} \equiv \mathrm{C}-\mathrm{R}^{\prime}\right)$ are highly reactive in the solid state [1]. Among these compounds, the symmetrical diacetylene 2,4 hexadiynylene bis(p-toluene sulfonate) named pTS undergoes topochemical polymerization in the crystalline state by exposure to radiation (UV, $\gamma, \mathrm{RX} . .$. ) or by thermal annealing [1]. A large size monocrystal of hydrogenated or deuterated pTS can be converted into fully polymerized crystal. It is possible to obtain partially polymerized crystals at any controlled polymer content $x$ between $x=0$ (monomer) [2] and $x=1$ (polymer) because polymerization kinetics by isothermal annealing are easy to obtain. Hereafter mixed monomer-polymer crystals will be referred as $\mathrm{M}_{1-x} \mathrm{P}_{x}$.

When going from monomer to polymer, the main modification is the transformation of "intermolecular Van der Waals" bonds to "covalent" bonds along the polymerization axis

\footnotetext{
$\left({ }^{*}\right)$ Author for correspondence

$\left({ }^{* *}\right)$ URA au CNRS $n^{\circ} 804$
}

(C) Les Éditions de Physique 1997 
b [3]. This structural change is associated to the rotation of the diacetylene backbone around an axis perpendicular to the stacking axis $b$. In the case of pTS-D (fully deuterated pTS where $R=R^{\prime}$ is $-C D_{2}-O-S_{2}-C_{6} D_{4}-C D_{3}$ ) this rotation is related to the decreasing of $b$ parameter from 5.15 to $4.91 \AA$ [4]. Secondary effects are also observed, as small rotations of the side groups [3].

Since the first work of Wegner on the solid-state polymerization in 1969, many studies have been devoted to this subject [5]. Structural studies has been performed by X-ray and elastic neutron scattering in pTS-H (hydrogenated pTS) [6-8].

Indeed, as in pTS-H [9-11], structural phase transitions occur in both monomer and polymer pTS-D crystals. In the fully polymerized monocrystal of pTS-D, an antiferroelectric phase transition occurs at $T_{\mathrm{c}}=190 \mathrm{~K}[12]$. From the high temperature phase (monoclinic $\mathrm{P} 2_{1} / \mathbf{n}$; $Z=2$ ) the transition yields a low temperature phase (monoclinic $\mathrm{P} 22_{1} / \mathrm{c} ; Z=4$ ) with a doubling of the unit cell along the a cristallographic axis. In the pure monomer an intermediate incommensurate phase takes place [13]: the incommensurate and lock-in phase transitions occur respectively at $T_{\mathrm{c}}=195 \mathrm{~K}$ and $T_{1}=155 \mathrm{~K}$ [4] in pTS-D. The high and low temperature phases of pTS monomer are isomorphous to the high and low temperature phases of the polymer [6-8]. These instabilities are both associated with motions of the lateral groups [8,12].

The possibility to obtain mixed monomer-polymer crystals at any controlled polymer content gives the opportunity to study a structural phase transition in a $M_{1-x} P_{x}$ system where $M$ is the monomer and $\mathrm{P}$ is the polymer. Among the mixed systems the originality of the monomerpolymer crystals consists, first in the highly anisotropic disorder induced by polymerization which occurs along $\mathbf{b}$ axis, and second in the fact that the structural phase transitions, which take place in both monomer and polymer crystals, have a similar behavior.

Microcalorimetric study of pTS-H has shown the progressive smearing of the monomer transition as a function of increasing polymer content: the thermal anomalies characteristic of the transitions totally disappear in the $[0.1-0.95]$ range of polymer content $x$. The calorimetric study of $\mathrm{M}_{1-x} \mathrm{P}_{x}$ crystals of $\mathrm{pTS}-\mathrm{D}$ does not show thermal anomaly in the $[0.1-0.6] x$ range [14]. X-rays measurements have been performed with precession method in $\mathrm{M}_{1-x} \mathrm{P}_{x}$ crystals of pTS-H [15]. They showed the existence of superstructure peaks, due to the cell doubling along the a crystallographic axis at low temperature and at every polymer content. The authors nevertheless report a strong increase of the widths of these peaks from $x \cong 0.1$ to $x \cong 0.95$. On the contrary, the polymerization has no effect on the Bragg peaks characteristic of the high temperature phase: their widths remain of the order of the value of the resolution function. The authors conclude that the crystallographic long range order is preserved at high temperature and at every polymer content $x$; it is not the case for the reorientations of the lateral groups at low temperature, and thus for the order parameter $\eta$.

In their study by neutron elastic scattering of pTS-D crystals, Grimm et al. [16] have not obtained the same results. They observed a strong decrease of the intensities of the Bragg reflections. They mentioned that this effect is due to the strong increase of the "mosaic" width indicating the presence of stress in the crystal. In addition they reported the decreasing of b parameter as a function of the polymer content. A (020) Bragg peak characteristic of the monomer vanishes in the background from $x=0$ to $x=0.4$; from $x=0.4$ to $x=1.0$, a new (020) Bragg peak characteristic of the polymer increases. At $x=0.4$, there is a coexistence of the two (020) Bragg peaks characteristic of the monomer and the polymer. These results are in total disagreement with the ones obtained by Robin et al. [13] for pTS-H by X-rays scattering: the authors reported a continuous evolution of the $\mathbf{b}$ parameter when going from $x=0$ to $x=1.0$. Grimm et al. [16] have also reported a steep variation of the superlattice intensity for $x=0.13\left(T_{\mathrm{c}}=166 \mathrm{~K}\right)$ and $x=0.19$ whereas a smearing of the phase transition up to $T=200 \mathrm{~K}$ for $x=0.6$ is observed. 
We have already performed some experiments on pTS-D crystals by using local methods (D-NMR [17], Raman scattering studies of pTS-D polymer high frequency modes [18]), as bulk (calorimetry [14]) and collective (neutron scattering and Raman scattering on low frequency modes of pTS-D monomer [4,19] and polymer [12] crystals) methods. In this paper we present elastic neutron scattering results on $\mathrm{M}_{1-x} \mathrm{P}_{x}$ crystals of $\mathrm{pTS}-\mathrm{D}$. Section 2 is devoted to experimental aspects. In Section 3 we show results obtained on the evolution of pTS-D Bragg peaks. In Section 4 we present the study of superstructure peaks. The phase diagram of pTS-D is determined for different polymer contents from 0 to 1 . Section 5 is the discussion.

\section{Experimental}

2.1. Synthesis, Crystal Growth and Polymerization. - $\mathrm{M}_{1-x} \mathrm{P}_{x}$ mixed crystals of pTS-D which have been investigated in our neutron scattering study have been synthesized and grown by one of us (M. Bertault). The synthesis of fully deuterated compound is described elsewhere [14]. Perdeuteration is necessary in order to reduce incoherent neutron scattering during coherent neutron scattering experiments. Single crystals of monomer were grown by slow evaporation at $4{ }^{\circ} \mathrm{C}$ from a solution of pTS-D in acetone under a controlled flow of nitrogen gas. In order to obtain homogeneous polymerization $[13,20]$ (randomly distributed chains in the monomer matrix) and good homogeneity of the polymer content in all the crystal, partially polymerized crystals were obtained by thermal annealing at $333 \mathrm{~K}$ in a thermostatically controlled bath; the time of the polymerization at a determined polymer content is deduced from isothermal polymerization kinetics of a small part of the same crystal at the same temperature in a DSC calorimeter [2]. Two tests were performed to control the polymer content $x$ : first. measurement of the residual polymer mass after dissolution of the monomer in a solution of warm acetone and after filtration; second, measurements of the $\mathbf{b}$ parameter by neutron scattering and their comparison to previously data measured on pTS-D crystals (see Sect. 3). We also performed a D-NMR study on the crystal at $x=0.6$ : it enables us to check the polymer content [17]. As the $b$ parameter varies too slowly from $x=0.8$ to $x=1.0$, the measure of $b$ cannot be used to determine $x$ in this range of polymer content. D-NMR study [17] enables us to correct the $x$ value for the named "polymer" pTS-D crystal of our previous study [12]. A value of $x=0.95$ was found instead of $x=1.0$. This is the reason why we have performed an additional neutron scattering study with a new pTS-D polymer crystal with a right value of $x=1.0$. For these studies, single crystals of pTS-D had volumes varying approximately from 0.07 to $0.6 \mathrm{~cm}^{3}$. During the polymerization the crystal has been placed in a thermostatically controlled bath with a temperature stability on the order of $0.1 \mathrm{~K}$. Thus, we did not use in situ polymerization because:

1) displex close cycle refrigerator does not allow to obtain a stabilized temperature of $T=60^{\circ} \mathrm{C}$;

2) we have verified that the contacts, via glue to metallic sample holder, induce temperature gradients in the crystals and then produce important macroscopic polymerization inhomogeneities in the crystal. Small temperature differences between the metallic contacts and the helium exchange gas cannot be neglected for the polymerization of diacetylenes.

2.2. Neutron Scattering. -- The experiments were performed at Orphée Reactor in Laboratoire Léon Brillouin (CEN Saclay, France), on the triple axis spectrometer 4F 1 installed on a cold neutron source. We used fixed incident wave vectors of $k_{\imath}=2.662 \AA^{-1}\left(E_{\mathrm{i}}=14.7 \mathrm{meV}\right)$ and $k_{2}=1.54 \AA^{-1}\left(E_{\mathrm{i}}=4.7 \mathrm{meV}\right)$, with respectively pyrolytic graphite and nitrogen cooled berylium used as filters to remove subharmonic wavelengths and $\left(40^{\prime}, 40^{\prime}\right)$ collimating elements (for the $x=0.015$ crystal, incident wave vector of $k_{\mathbf{i}}=1.4 \AA^{-1}$ was also used). The samples 
were investigated in $\left(\mathbf{a}^{*}, \mathbf{b}^{*}\right)$ plane for all the experiments. Full width half maximum (FWHM) of the $Q$-resolution was around FWHM $=0.015 \AA^{-1}$ and $F W H M=0.040 \AA^{-1}$ respectively for $k_{\mathrm{i}}=1.54 \AA^{-1}$ and $k_{\mathrm{i}}=2.662 \AA^{-1}$ (some more details are added in Figs. in part 4 ). We used a Displex close-cycle refrigerator ensuring a temperature stability of about $0.1 \mathrm{~K}$. The refinements were performed with fitting procedure of Laboratoire Léon Brillouin [21] using convolution of the chosen functions by the resolution function [22].

\section{Evolution of the Bragg Peaks}

Figure 1 shows the values of mosaïc width for the pTS-D crystals. The average value of mosaïc width is roughly equal to $0.5 \mathrm{deg}$. In the case of the pTS-D sample at $x=0.4$, the slightly higher value of mosaïc width is due to the initial quality of the crystal and not to the polymerization process itself.

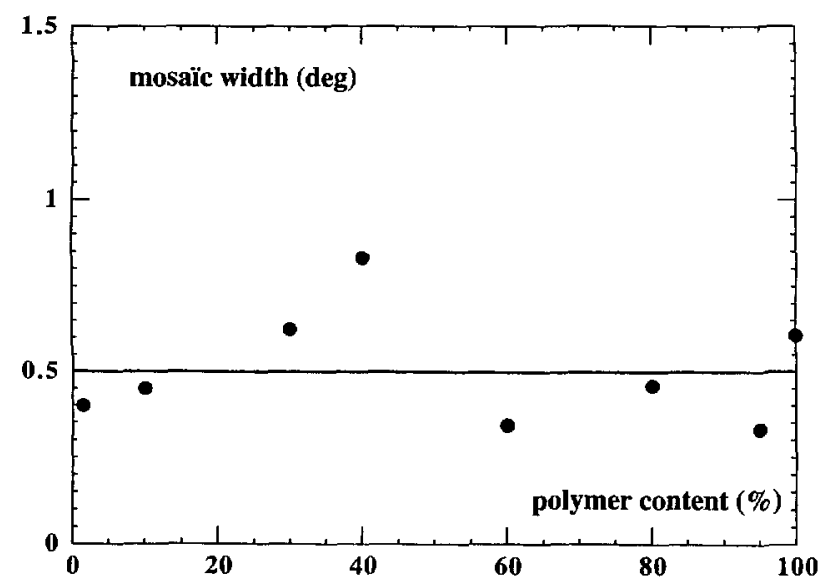

Fig. 1. - Evolution of the mosaï width in $\mathrm{M}_{1-x} \mathrm{P}_{x}$ mixed crystals of $\mathrm{pTS}-\mathrm{D}$ as a function of polymer content.

We have measured the evolution of the $(0,2,0)$ Bragg peaks as a function of the temperature and of the polymer content $x$. The variation of the $b$ parameter at $T=270 \mathrm{~K}$ as a function of $x$ (Fig. 2) corresponds to the one observed by Aimé [23]: this result confirms the estimation of the polymer content values as we mentioned before (Sect. 2.1).

Figure 3 shows the experimental shape of the $(0,2,0)$ Bragg peaks for $x=0.1,0.3,0.8,1.0$ at $T=270 \mathrm{~K}$. The same profile is obtained for all $x$ values except for $x=0.3$. The $(0,2,0)$ Bragg peak in the crystal at this polymer content has an asymmetric profile along $\mathbf{b}^{*}$ and is broader (see for comparison the shape obtained for $x=0.1$ and $x=0.8$ ) whereas the $(5,0,0)$ reflexion remains symmetrical and keeps almost the same width. The profiles of these Bragg peaks are almost temperature independent in every direction, $\mathbf{a}^{*}$ or $\mathbf{b}^{*}$. Because of the small $b$ parameter, and hence the large value of $\mathbf{b}^{*}$, it was not possible to measure intensity of the $(0,4,0)$ peak at $k_{1}=1.54 \AA^{-1}$ (the $(0, k, 0)$ reflexions with $k=2 n+1$ are absent) in order to obtain the evolution of the profiles as a function of $\mathbf{Q}$. 


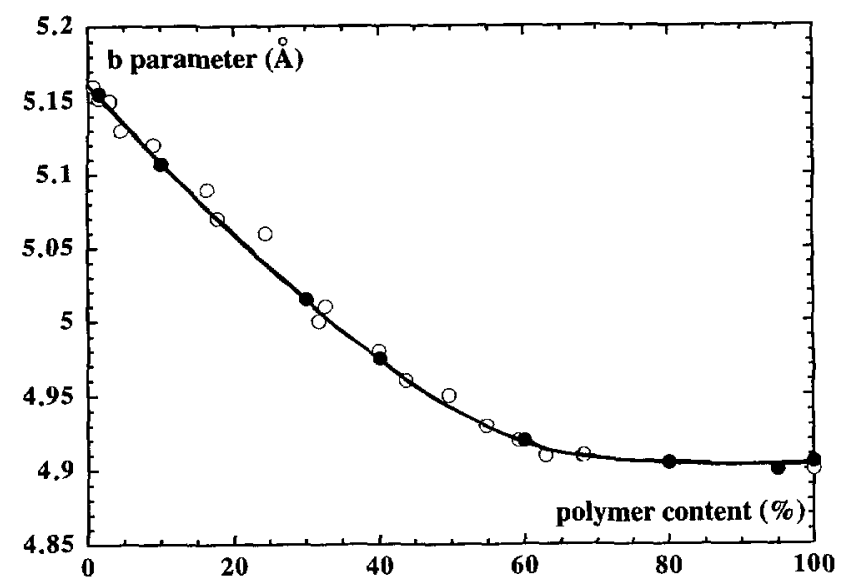

Fig. 2. - Evolution of $\mathbf{b}$ parameter value in pTS-D as a function of the polymer content: open circles and line from Aimé $[23]$ at $280 \mathrm{~K}$. The full circles, deduced from $b$ measurement at $T=270 \mathrm{~K}$, give the polymer content of the samples studied in this work by neutron scattering: $x=0.015, x=0.1$, $x=0.3, x=0.4, x=0.6, x=0.8, x=0.95$ and $x=1.0$.

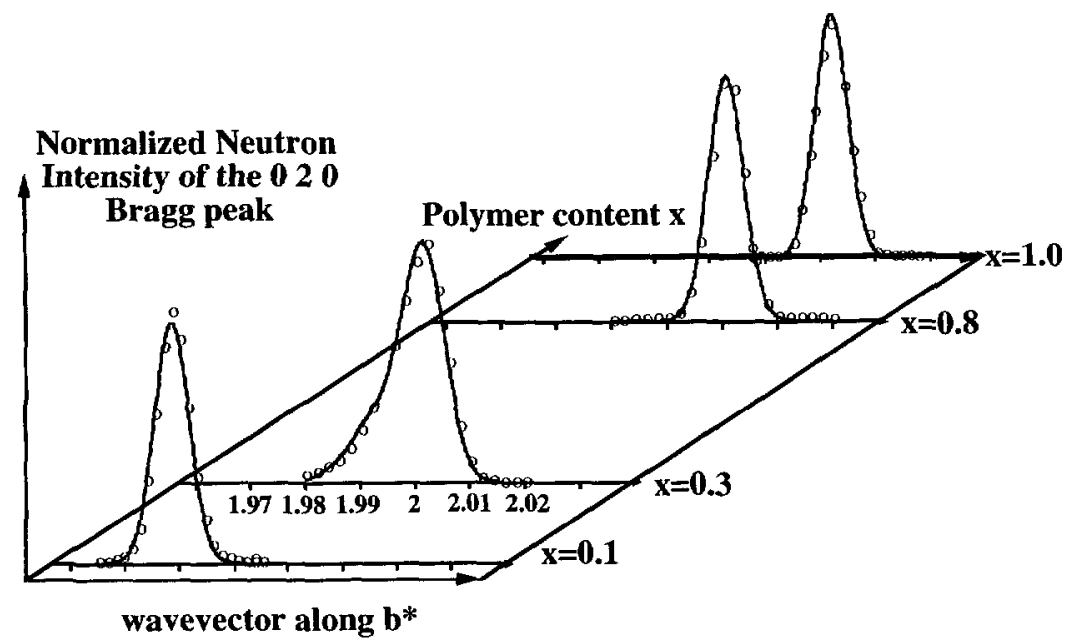

Fig. 3. - Evolution of the $(0,2,0)$ Bragg peak in $\mathrm{M}_{1-x} \mathrm{P}_{x}$ mixed crystals of pTS-D at $120 \mathrm{~K}$ as a function of the polymer content $\left(k_{1}=1.54 \AA^{-1}\right)$. An effect of the disorder on the width can be observed for the pTS-D crystal at $x=0.3$.

\section{Evolution of the Superstructure Peaks}

4.1. PTS-D CRYSTAL AT $x=0.015$. - Satellites characteristic of the incommensurate phase were observed in this pTS-D crystal at very low polymer content. Figure 4 shows the evolution of the incommensurate parameter $\delta$ as a function of temperature at $x=0.015\left(k_{\mathrm{i}}=1.4 \AA^{-1}\right)$. The lock-in transition is located at $T_{1}=155 \mathrm{~K}$. $T_{1}$ was found equal to $164 \mathrm{~K}$ in the crystal at $x=0.1$. The widths of the satellites along $\mathbf{a}^{*}$ and $\mathbf{b}^{*}$ are larger than the widths of the superstructure peaks below $T_{1}$. 


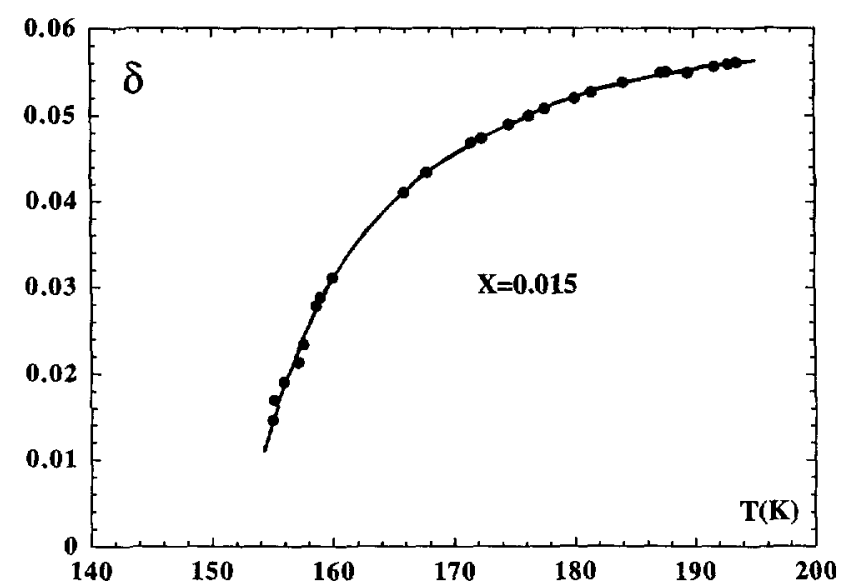

Fig. 4. - Evolution of the incommensurate parameter $\delta$ as a function of temperature for the pTS-D crystal at $x=0.015$. The drawn line is a guide to the eye.

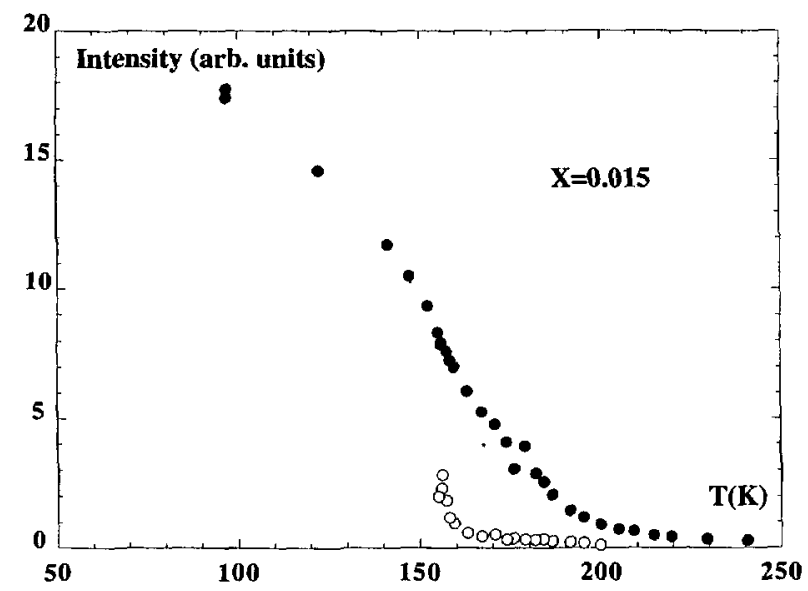

Fig. 5. -- Integrated intensity of the $(6.5,1,0)$ superstructure peak as a function of the temperature in the pTS-D crystal at $x=0.015$ : critical scattering at $(6.5,1,0)$ (open circles) and critical scattering at $(6.5,1,0)$ plus integrated intensity of incommensurate satellites $(6.5,1 \pm \delta, 0)$ (full circles).

The integrated intensities of the $(6.5,1 \pm \delta, 0)$ satellites and of the $(6.5,1,0)$ superstructure peaks characteristic of the low temperature phase $\left(T<T_{1}\right)$ were studied at $k_{\mathrm{i}}=2.662 \AA^{-1}$ as a function of temperature in the crystal at $x=0.015$ (Fig. 5 ). We must note that the satellites and the superstructure peaks coexist from roughly $T_{1}=155 \mathrm{~K}$ to $T=165 \mathrm{~K}$. We consider that, as the modulation along $\mathbf{b}^{*}$ of the cell doubling along $\mathbf{a}^{*}$ is weak in the incommensurate phase $(\delta<0.06)$, it is possible to add the contributions of the central part and of the satellites over the whole temperature range. This variation can be compared to the one observed in pTS-D crystals where no satellites are observed (see Sect. 4.3). A high transition temperature $T_{\mathrm{c}}$ for the cell doubling along $\mathbf{a}^{*}$ is then found equal to about $195 \mathrm{~K}$ in the crystal at $x=0.015$. 

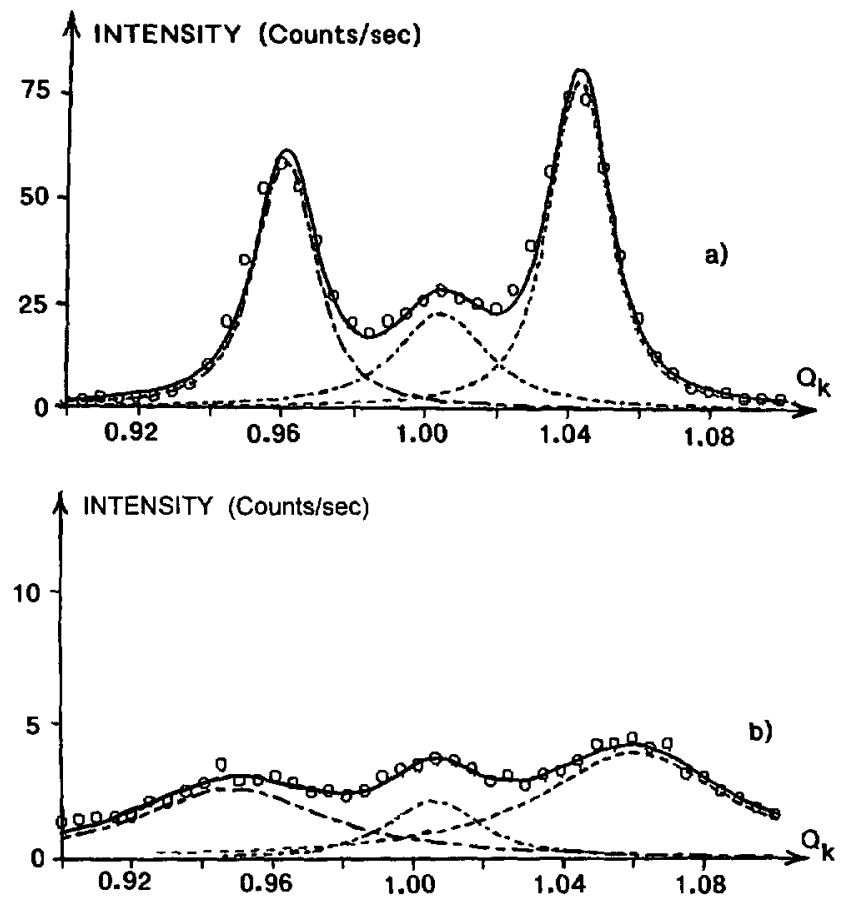

Fig. 6. - Satellites and superstructure peaks for the crystal at $x=0.1$, measured along the $\mathbf{b}^{*}$ axis at $T=174 \mathrm{~K}(\mathrm{a})$ and $T=202 \mathrm{~K}(\mathrm{~b})$ at $k_{1}=1.54 \AA^{-1}$

4.2. PTS-D CRYSTAL AT $x=0.1$. - Satellites and superstructure peaks coexist from $T=155 \mathrm{~K}$ to at least $220 \mathrm{~K}$ in this mixed pTS-D crystal (above $T=220 \mathrm{~K}$ the intensity is very small). It is difficult to determine a well defined lock-in transition as in the pTS-D crystal at $x=0.015$. Satellites are clearly observed down to $T=168 \mathrm{~K}$, between $T=155 \mathrm{~K}$ and $T=168 \mathrm{~K}$; satellites and superstructure peaks are very close ( $\delta$ is small). The widths of the superstructure peaks along $\mathbf{a}^{*}$ and $\mathbf{b}^{*}$ and the widths of the satellites increase at $T^{\mathrm{O}}=153 \mathrm{~K}$ ( $T^{\mathrm{O}}$ is defined as the temperature of ordering). The most interesting result is that the width of the superstructure peak along $\mathbf{b}^{*}$ does not change (Fig. 6) above $T^{\mathrm{O}}$ whereas this width along $\mathbf{a}^{*}$ increases continuously and its intensity decreases. At the same time the width of the satellites along $\mathbf{b}^{*}$ continuously increases (Fig. 6). When the contributions of the central part and of the satellites are added, a high transition temperature $T_{\mathrm{c}}$ for the cell doubling along $\mathbf{a}^{*}$ is then found equal to about $195 \mathrm{~K}$.

4.3. PTS-D CRYSTALS AT $x=0.3,0.4,0.6$ AND 0.8 . - Satellites are no more observed in these mixed pTS-D crystals. The effect of the polymerization on the evolution of the superstructure integrated intensity as a function of the temperature is reported in Figure 7: the variation of the intensity of the $(5.5,2,0)$ reflexion $\left(k_{\mathrm{i}}=2.662 \AA^{-1}\right)$ is showed in the crystals at $x=0.3, x=0.4, x=0.6$ and $x=0.8$. The intensities have been drawn on the same scale because crystals of different sizes were used: it is not possible to give a quantitative description of the intensity as a function of $x$. The evolution of the intensities always appears continuous with a decrease up to $T_{\mathrm{c}}$ located in the $[190-210 \mathrm{~K}]$ range. Above $200 \mathrm{~K}$ a strong diffuse scattering is still observed in the disordered crystals, particularly in the crystal at $x=0.40$. There is no saturation of the scattered intensity down to very low temperature. 


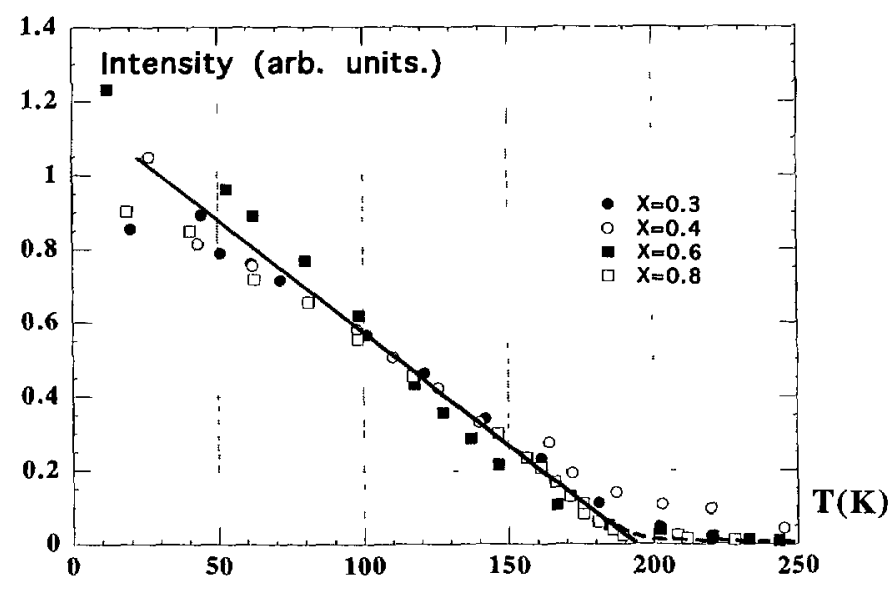

Fig. 7. - Evolution of the integrated intensity of the $(5.5,2,0)$ superstructure reflection as a function of the temperature in pTS-D crystals at $x=0.3,0.4,0.6$ and $0.8\left(k_{1}=2.662 \AA^{-1}\right)$.

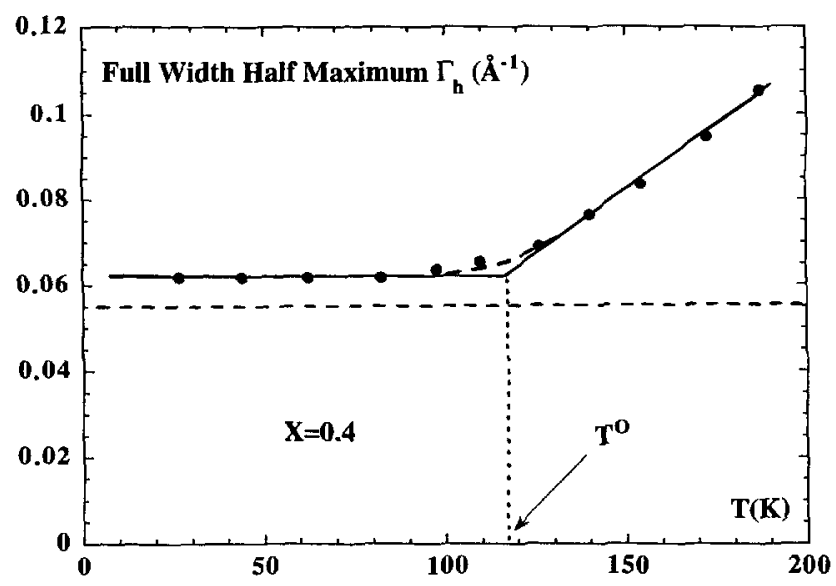

Fig. 8. - Evolution of the FWHM of $\mathbf{Q}_{\mathrm{h}}=(\xi, 2,0)$ profiles in the pTS-D crystal at $x=0.4$ as a function of the temperature $\left(k_{1}=2.662 \AA^{-1}\right)$. Dotted line shows the resolution.

Figure 8 shows the evolution of the full width at half maximum along $\mathbf{a}^{*}$ as a function of temperature in the crystals at $x=0.4\left(k_{1}=2.662 \AA^{-1}\right)$. The temperature $T^{\mathrm{O}}=115 \mathrm{~K}$ corresponds to the increase of the width along $\mathbf{a}^{*}$. In the crystals at $x=0.3,0.6$ and $0.8, T^{\mathrm{O}}$ is equal to respectively $120 \mathrm{~K}, 120 \mathrm{~K}$ and $155 \mathrm{~K}$. Along $\mathbf{b}^{*}$, the behaviour is different. The full width at half maximum in the crystal at $x=0.3$ has a very slow variation as a function of temperature: it is shown in Figure $9 \mathrm{a}$ at $k_{\mathrm{i}}=2.662 \AA^{-1}$ With an improved $Q$-resolution $\left(k_{1}=1.54 \AA^{-1}\right)$, a small increase of the width along $\mathbf{b}^{*}$ above $T^{\mathrm{O}}$ can be detected (Fig. $9 \mathrm{~b}$ ). The same results were obtained in the crystals at $x=0.3$ and $x=0.6$ with $k_{\mathrm{i}}=2.662 \AA^{-1}$ In the crystal at $x=0.8$, the evolution of the width along $\mathbf{b}^{*}$ (Fig. 10) is stronger than in the crystals at $x=0.1$ (superstructure peak), $x=0.3, x=0.4$ and $x=0.6$ but remains smaller than along $\mathbf{a}^{*}$. 

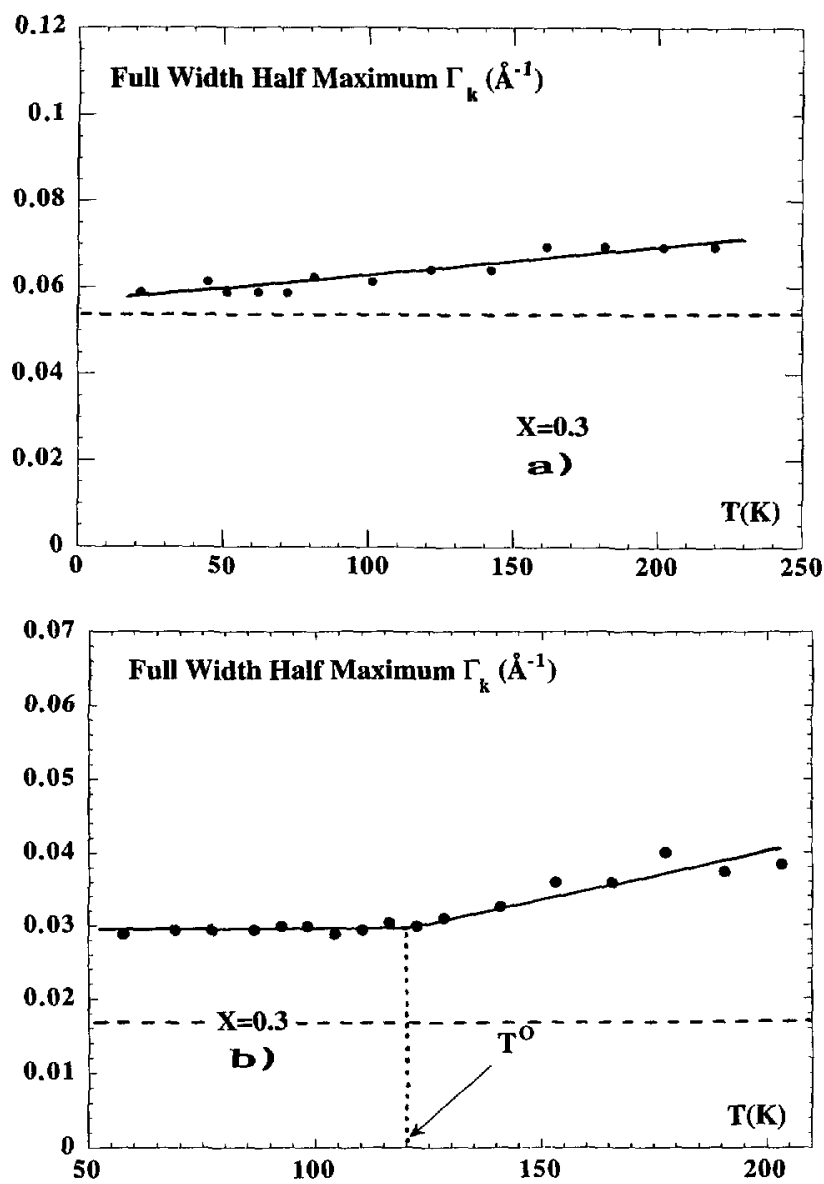

Fig. 9. - a) Evolution of the FWHM of $\mathrm{Q}_{k}=(5.5, \xi, 0)$ profiles in the pTS-D crystal at $x=0.3$ as a function of the temperature $\left(k_{1}=2.662 \AA^{-1}\right)$. Dotted line shows the resolution. b) Evolution of the FWHM of $\mathbf{Q}_{\mathrm{k}}=(3.5, \xi, 0)$ profiles in the pTS-D crystal at $x=0.3$ as a function of the temperature $\left(k_{1}=1.54 \AA^{-1}\right)$. Dotted line shows the resolution.

4.4. PTS-D PHASE DIAGRAM. - The neutron scattering $\mathrm{pTS}-\mathrm{D}$ phase diagram is constructed from $T_{\mathrm{c}}$ and $T^{\mathrm{O}}$ values deduced from the studies above mentioned (Fig. 11). In the pTS-D crystal at $x=0.95$, two temperatures $T^{\mathrm{O}}=182 \mathrm{~K}$ and $T_{\mathrm{c}}=190 \mathrm{~K}$ can also be defined from the width and integrated intensity evolutions as a function of temperature (see Figs. 5 and 6 of Ref. [12] in analogy to the ones defined in Sect. 4.3).

\section{Discussion}

5.1. Evolution of the Bragg Peaks. - Grimm et al. [16] mentioned that the decrease of the Bragg intensities was mainly due to the increase of the "mosaic" width, which is the result of the stress appeared in the crystal. We believe that in fact this is probably due to the in situ polymerization used by Grimm et al. for their samples. We did not observed such an increase of the mosaic width in the mixed monomer-polymer crystals obtained by using 

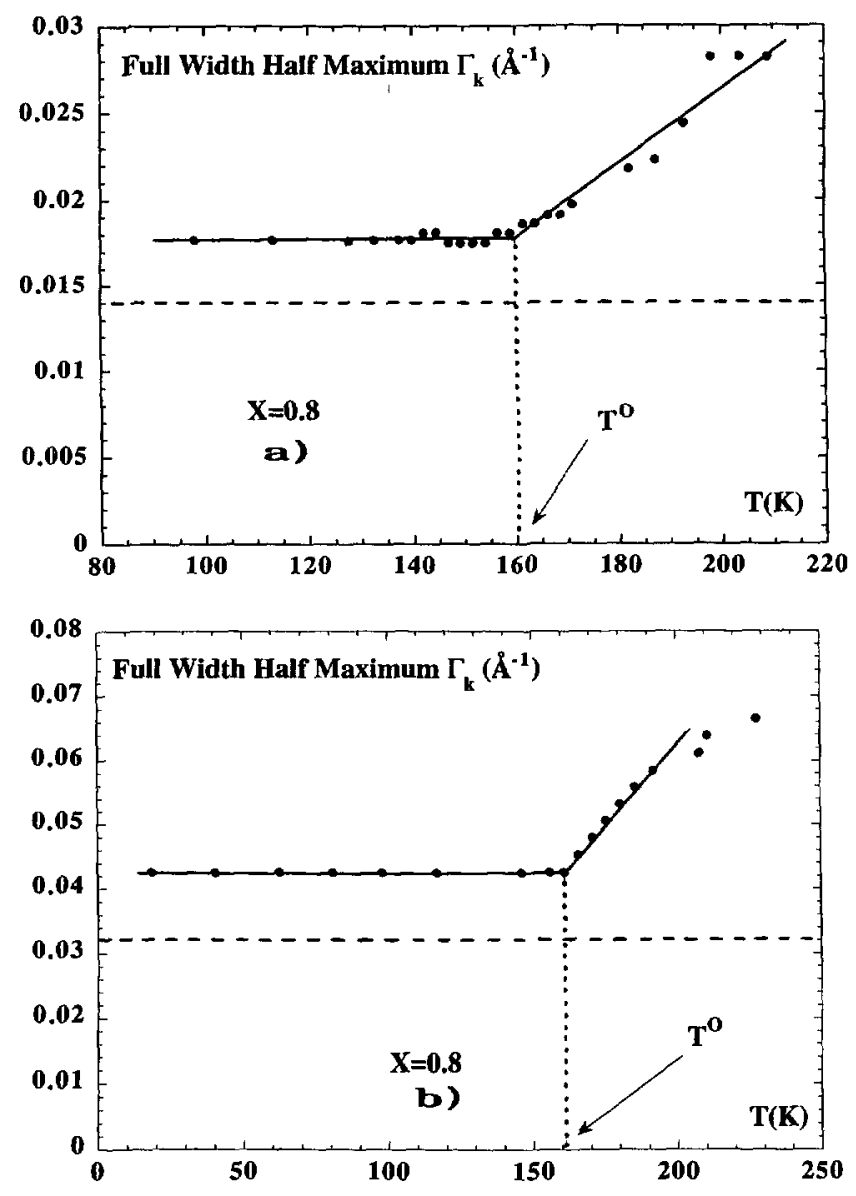

Fig. 10. - a) Evolution of the FWHM of $\mathbf{Q}_{\mathrm{k}}=(3.5, \xi, 0)$ profiles in the pTS-D crystal at $x=0.8$ as a function of the temperature $\left(k_{1}=1.54 \AA^{-1}\right)$. Dotted line shows the resolution. b) Evolution of the FWHM of $\mathbf{Q}_{\mathrm{k}}=(5.5, \xi, 0)$ profiles in the pTS-D crystal at $x=0.8$ as a function of the temperature $\left(k_{1}=2.662 \AA^{-1}\right)$. Dotted line shows the resolution.

a thermostatically controlled water bath. A temperature gradient of $1 \mathrm{~K}$ induces, after a few hours of heating at around $333 \mathrm{~K}$, a strong variation of the polymer content in the bulk as we have verified (see part 2.1). A part of the crystal heated at $T=59^{\circ} \mathrm{C}$ could be still in the induction period at $x \leq 0.2$ whereas another part of the crystal heated at $T=60^{\circ} \mathrm{C}$ could have reached the autocatalytic period at $0.2<x<0.8$.

5.2. Evolution of the Superstructure Peaks. - Our results are inconsistent with those of Grimm et al. [16] which show in the crystal at $x=0.13$ and at $x=0.19$ a strong dependence of the superstructure intensity as a function of the temperature around 160-170 K. However these two degrees of polymerization have been calculated from the curve giving the dependence of the $b$ parameter at $120 \mathrm{~K}$ as a function of polymer content when polymerization is made by X-rays for pTS-H [24]. We know at present that the evolution of $b$ value as a function of the polymer content in pTS-D crystals is different than in pTS-H. So the two $b$ 


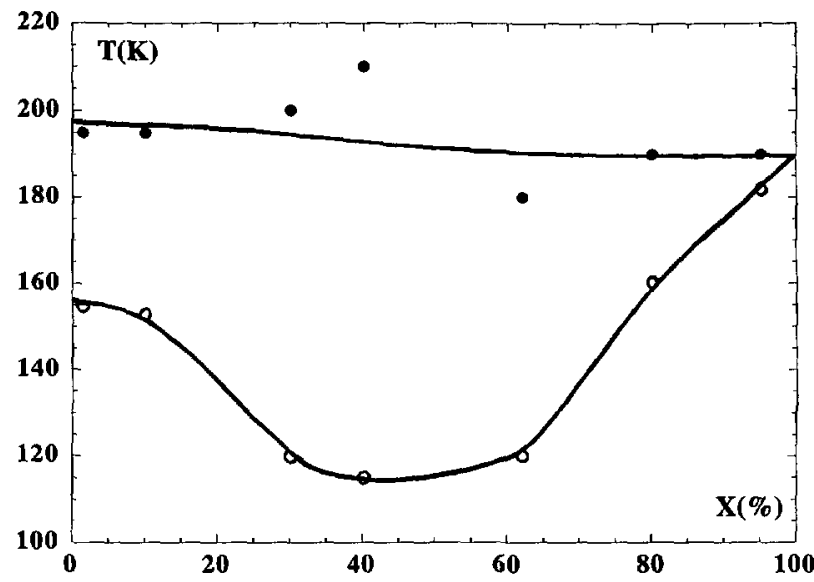

Fig. 11. - Phase diagram for the $\mathrm{M}_{1-x} \mathrm{P}_{x}$ system of pTS-D mixed crystal in the $] 0,1[x$ range obtained by neutron scattering. $T^{\mathrm{O}}$ (open circles) given by neutron scattering is the temperature which corresponds to the clamping of the correlation length at low temperature and $T_{c}$ (full circles) to the decrease of the integrated intensity. Dotted lines are guide to the eye.

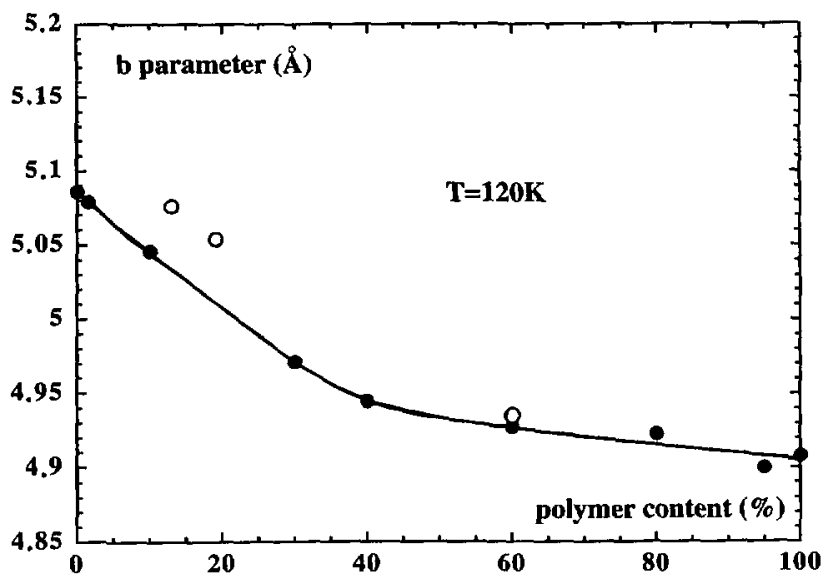

Fig. 12. - Evolution of $\mathbf{b}$ parameter value in pTS-D mixed crystal as a function of the polymer content at $120 \mathrm{~K}$ (from Grimm et al. [16,23]: open circles). The full circles, deduced from $b$ measurement, give the polymer content of the samples studied in this work by neutron scattering: $x=0.015, x=0.1$, $x=0.3, x=0.4, x=0.6, x=0.8, x=0.95$ and $x=1.0$. The value obtained for the crystal at $x=0$ was measured by Aimé et al. [8].

values measured at $120 \mathrm{~K}$ by Grimm et al. in mixed crystals of pTS-D correspond to $x=0.13$ and $x=0.19$ when these polymer contents are determined from the conversion curve for pTS-H established in reference [24]. The two same $b$ values measured at $120 \mathrm{~K}$ in our pTS-D mixed crystals correspond in fact respectively to $x=0.015$ and $x=0.1$ (Fig. 12). The two previous mixed crystals studied by Grimm et al. should have presented an incommensurate phase (see Sects. 4.1 and 4.2). Intensity evolution given by Grimm et al. (Fig. 4 of Ref. [16]) is indeed the same that we obtain in these two crystals when we do not take into account the intensity 


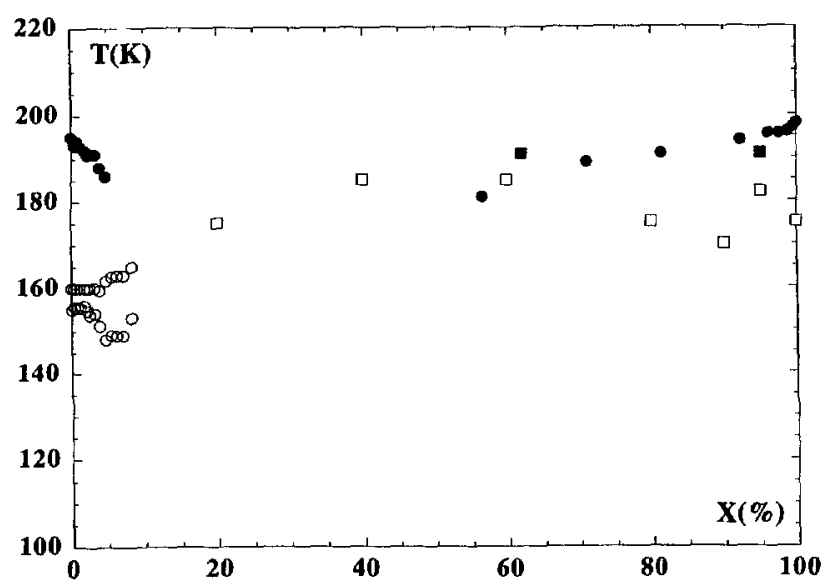

Fig. 13. - Phase diagram for the $M_{1-x} P_{x}$ system of pTS-D mixed crystal in the $] 0,1[x$ range obtained by calorimetric measurements (full and open circles) [14], Raman scattering (open squares) [18] and D-NMR (full squares) [17].

of satellites in the incommensurate phase. In order to determine $T_{\mathrm{c}}$, the addition of the two intensities (superstructure peaks and satellites) and not the intensity of only the superstructure peak must be considered. In this case the transition appears continuous as in mixed pTS-D crystals at $0.3<x<0.8$ (Fig. 7). It is not possible, as Grimm et al, to conclude that the difference between the results obtained for the crystals which are in the induction period (and present an incommensurate phase) and crystals being in the autocatalytic region corresponds to a smearing out of the transition. It is the evolution of the widths of superstructure peaks and satellites which will give the most important informations on the influence of disorder on the structural properties of mixed crystals.

The phase transition for both pure pTS-D monomer and pure pTS-D polymer crystals is antiferroelectric with a doubling of the a parameter: the non-polar lattice existing above $T_{\mathrm{c}}$ is divided into two polar sublattices below $T_{\mathrm{c}}$ with non-zero spontaneous polarizations lying along direction of $\mathbf{b}$ axis and alternatively oriented in opposite directions along a axis [25]. pTS-D monomer and polymer present many similarities but the signatures of the phase transitions as viewed by calorimetry disappear in mixed monomer-polymer crystals of pTS-D (Fig. 13) [14]. As pTS-D monomer and polymer have the same critical temperature $T_{\mathrm{c}}$, the same order parameter (side-groups reorientation) and the same soft mode evolution $[4,12,19]$. other explanations have to be found to explain the disorder observed at intermediate polymer content. At first, the main difference between pTS-D monomer and polymer is the modulation along the $\mathbf{b}$ axis of the cell doubling along the a axis in pTS-D monomer. This is shown in particular for the compound at $x=0.1$ (Sect. 4.2) where satellites and superstructure peaks coexist over a temperature range corresponding roughly to the incommensurate phase of the monomer. A frustration may result from the presence of neighbouring columns of different types extending along the $\mathbf{b}$ axis. Along columns made of monomer units, side-groups have a tendency to adopt a modulated orientation whereas this modulation is not allowed along polymer chains. It may explain why the long range order is suppressed only in an intermediate temperature range above $T^{\mathrm{O}}$ (Fig. 11). The second important point is that the intensity of the superstructure peaks does not vanish in the temperature range between $T^{\mathrm{O}}$ and $T_{\mathrm{c}}$ : this is related to the fact that the correlations along the $\mathbf{b}$ axis remain strong. One may make the hypothesis that 
the superstructure peaks are related to the ordering along the polymer chains. Raman scattering study of pTS-D polymer chains [18] shows indeed that the cell doubling along the polymer chains is observed even in a crystal at $x=0.2$. On the contrary, satellites are strongly affected by the disorder (Sect. 4.2): they may then be attributed to the remainder of the monomer lattice. The correlations along the a axis are weak both for the satellites and the superstructure peaks: this is consistent with the fact that the polymer chains are randomly distributed in the $(\mathbf{a}, \mathbf{c})$ plane in the monomer matrix. A physical interpretation of the pTS-H phase diagram was done by Pouget [26]. There is a pinning of the domain walls near the polymer chain ends around the lock-in transition in pTS-H monomer crystals because there is a coincidence of the length equal to $1 / 2 \delta$ and the length of the polymer chains. In pTS-D, as the polymer chains are probably longer $[14,27,28]$, such effect is weaker.

As a conclusion, we may say that the phase diagram of mixed pTS-D monomer-polymer crystals is interesting from two points of view: first, the disorder arises probably from a frustrated incommensurate modulation and second, the influence of extended defects (polymer chains) appears as a determining factor in the observed phenomena.

\section{References}

[1] Wegner G., Z. Naturforschung 24b (1969) 824.

[2] Bertault M., Schott M., Brienne M.J. and Collet A., Chem. Phys. 85 (1984) 481.

[3] Enkelmann V. and Wegner G., Angev. Chem. Int. Ed. Engl. 16 (1977) 416.

[4] Even J., Toudic B., Bertault M., Cailleau H. and Moussa F., Phys. Rev. B 52 (1995) 7142.

[5] See for example: Polydiacetylenes, NATO ASI series, D. Bloor and R.R. Chance, Eds. (Martinus Nijhoff Publishers 1985).

[6] Van Kobelt D. and Paulus E.F., Acta Cryst. B30 (1974) 232.

[7] Enkelmann V., Acta Cryst. B33 (1977) 2842.

[8] Aime J.P., Lefebvre J., Bertault M., Schott M. and Williams J.O., J. Phys. France 43 (1982) 307.

[9] Bloor D., Ando D.J., Preston F.H. and Stevens G.C., Chem. Phys. Lett. 24 (1974) 407.

[10] Reimer B., Bassler H., Phys. Status Solıdi (B) 73 (1976) 709.

[11] Enkelmann V. and Wegner G., Makromol. Chem. 178 (1977) 635.

[12] Even J., Bertault M., Toudic B., Cailleau H., Fave J.L., Currat R. and Moussa F., Phys. Rev. B 49 (1994) 1602.

[13] Robin P., Pouget J.P.. Comes R. and Moradpour A., J. Phys. France 41 (1980) 415.

[14] Even J., Bertault M., Longeville S. and Collet A., J. Polym. Scr. B: Polym. Phys. 35 (1997) 789.

[15] Patillon J.N., Robin P., Albouy P.A., Pouget J.P. and Comes R., Mol. Cryst. Lıq. Cryst. 76 (1981) 297.

[16] Grimm H., Axe D. and Krohnke C., Phys. Rev. B 25 (1982) 1709.

[17] Longeville S., Etrillard J., Bertault M., Even J., Gallier J. and Toudic B., Mol. Cryst. Liq. Cryst. 277 (1996) 315.

[18] Longeville S., Bertault M., Even J., Fave J.L., Girard A. and Delugeard Y., Chem. Phys. 195 (1995) 371.

[19] Bertault M., Even J., Girard A. and Delugeard Y., Adv. Mater. Opt. Elec. 6 (1996) 317.

[20] Albouy P.A., Keller P. and Pouget J.P., J. Am. Chem. Soc. 104 (1982) 6556. 
[21] Hennion M., Hippert F. and Murani A.P., J. Phys. F 14 (1984) 489 (Appendix).

[22] Cooper M.J. and Nathans R., Acta Cryst. 23 (1967) 357.

[23] Aime J.P., Thesis, University Paris VII, France (1983).

[24] Enkelmann V., Leyrer R.J. and Wegner G., Makromol. Chem. 180 (1979) 1787.

[25] Nowak R., Sworakowski J., Kuchta B., Bertault M., Schott M., Jakubas R. and Kolodziej H.A., Chem. Phys. 104 (1986) 467.

[26] Pouget J.P., Europhys. Lett. 11 (1990) 645.

[27] Niederwald H. and Schwoerer M., Z. Naturforsch 38a (1983) 749.

[28] Gruner-Bauer P., Muller I., Dormann E. and Krohncke C., Makromol. Chem. 192 (1991) 1541. 